\title{
Risk Factors for Falls and Fragility Fractures in Community-Dwelling Seniors: A One-Year Prospective Study
}

\author{
Sacha Song, ,2, Joy C. MacDermid, $^{3,4}$ and Ruby Grewal ${ }^{3}$ \\ ${ }^{1}$ Schulich School of Medicine \& Dentistry, University of Western Ontario, London, ON, Canada N6A 5C1 \\ ${ }^{2}$ University of Windsor, Medical Education Building Room 1100, 401 Sunset Avenue, Windsor, ON, Canada N9B 3P4 \\ ${ }^{3}$ Division of Orthopedic Surgery, University of Western Ontario, Hand and Upper Limb Center, St. Joseph's Health Care, \\ 268 Grosvenor Street, London, ON, Canada N6A 4L6 \\ ${ }^{4}$ School of Rehabilitation Sciences, McMaster University, 1400 Main Street West, Hamilton, ON, Canada L8S 1C7
}

Correspondence should be addressed to Ruby Grewal; rgrewa@uwo.ca

Received 21 June 2013; Accepted 18 July 2013

Academic Editors: P. Czarnecki, E. E. Hansson, and H. Unalan

Copyright (C) 2013 Sacha Song et al. This is an open access article distributed under the Creative Commons Attribution License, which permits unrestricted use, distribution, and reproduction in any medium, provided the original work is properly cited.

Objective. To evaluate risk factors for falls and fragility fractures in healthy seniors. Methods. Assessing 50 ambulatory communitydwelling volunteers $\geq 65$ for demographics, BMI, bone mineral density (BMD) (DEXA), fracture risk (FRAX), balance (Biodex), fear of falling (Modified Falls Efficacy Scale (MFES)), and activity level (RAPA). One-year followup was done through phone interviews. Results. Most participants (17 males, 33 females; mean age 72.0 \pm 5.5 years) had normal BMD and were active with little to no fear of falling. Balance did not correlate with FRAX or fear of falling. Activity level did not correlate with FRAX, but the active group had less fear of falling. Most scored below age specific norms on balance testing. Fear of falling was not significantly different between genders but did correlate with FRAX, indicating that patients with higher fracture risk were also more afraid of falling. Individuals who fell after one year had increased fear of falling and decreased activity levels. Conclusions. Community-dwelling seniors with higher risk of future fractures were more afraid of falling. Although healthy and active, this cohort had poor balance compared to age matched norms. Further research on how to best assess fall risk and improve balance to prevent fractures is needed.

\section{Introduction}

Fractures resulting from a fall from standing height (fragility fractures) account for significant morbidity and health care expenses in older patients [1-3]. The 65-year and older age bracket is the fastest growing demographic around the world in both industrialized and developing countries [4, 5]. In industrialized countries, an estimated one-third of individuals $\geq 65$ experience at least one fall each year [6,7]. In 2008-2009, more than half of the cases of injurious falls were experienced by individuals $\geq 65$, and within this age group, falls were responsible for $74 \%$ of major injuries suffered and approximately $10 \%$ to $15 \%$ resulted in fracture [8].

Regardless of whether a fracture occurs, falls can lead to a reduced quality of life and a functional decline for the individual $[1,7]$. Falls in this age group are also more likely to result in longer and more expensive hospitalizations [1-3]. Annually, the cost to treat falls is $0.85 \%$ to $1.5 \%$ of the total health care expenditure $[2,3]$. Fractures are the most costly result of falls; Stevens et al. found that although fractures accounted for only $35 \%$ of injuries sustained from falls, they were responsible for $61 \%$ of related health care costs [2].

Since a fracture event is determined both by risk of falling and bone susceptibility, factors that adversely affect either predispose individuals for fracture. Balance impairments are a common cause of falls in the elderly population. Bone mineral density (BMD) has also been established as a risk factor for fall-related fractures. Fear of falling relates to an individual's perception of risk, past experiences, and attitudes about future falls. There is little research investigating the interrelationships between fear of falling, balance deficits, BMD, and estimated risk of fracture. Thus, it is unclear whether patients' stated views about their fear of future falls provide sufficient guidance for clinicians to take action or whether more detailed investigations are necessary.

One factor that has been shown in studies to be related to fall risk is activity level in older adults $[2,9,10]$. Decreased 
activity levels are believed to lead to functional decline, predisposing to falls $[2,9,10]$. In order to implement appropriate interventions to keep older adults active and to prevent functional decline, we need to understand what the current activity level of the community-dwelling population is so that we can assess their current risk of falls and fractures.

In order to prevent falls and their consequences, individuals who are at risk of falling need to be identified so that appropriate interventions and preventative measures can be put in place.

A better understanding of the role of balance, fear of falling, activity level, and BMD in fracture risk may lead to the development of more effective preventive measures that encompass multiple risk factors. As well, the incorporation of FRAX scores as a clinical tool in evaluating fracture risk will be considered. The purpose of this study was to assess risk factors for fragility fractures and correlations between these risk factors in a population of healthy volunteers aged $\geq 65$ living independently in the community. Risk factors assessed include fear of falling, activity level, balance, BMD, and FRAX scores. These individuals were then reassessed at the oneyear mark to gain a better understanding of how these risk factors actually influenced whether individuals suffered a fall or fragility fracture. As well, gender differences of these risk factors were also assessed.

\section{Methods}

2.1. Study Design. All study procedures were reviewed and approved by the Health Sciences Research Ethics Board at the University of Western Ontario for this prospective cohort study.

Fifty ambulatory subjects aged $\geq 65$ were recruited from the community (via poster advertisements). Individuals who were living in an institution, required the use of walking aids, had sustained a fragility fracture within the past year, or had a known balance disorder were excluded.

Participants completed a data collection form to determine their general health. Additional data collected included weight and height, which was used to calculate body mass index (BMI) as well as age and gender. The participant's fall and fracture history, family history of hip fracture, medical comorbidities, medications, and smoking and alcohol use were also collected in this survey. This information, along with $\mathrm{BMD}$, was then used to determine the participant's tenyear probability of osteoporotic and hip fracture using the Fracture Risk Assessment Tool (FRAX) [11-13].

FRAX is a tool that can help assess fracture risk. Launched by the World Health Organization (WHO) in 2008, FRAX is a web-based algorithm that calculates an individual's tenyear probability of both osteoporotic and hip fracture based on clinical information and BMD [11-13]. The information obtained from FRAX is meant to help guide clinicians in their treatment decisions but should be used in conjunction with clinical presentation and judgment [11-13]. In addition, FRAX has been calibrated for use in specific countries based on epidemiological data, making it a valid fracture model for specific populations $[12,13]$.
The Modified Falls Efficacy Scale (MFES) was administered at this time. The MFES is a reliable valid means of evaluating an individual's fear of falling by asking them to rate their confidence level in performing 14 different daily activities without falling (zero indicates no confidence, ten indicates complete confidence) [14]. The overall fear of falling score is determined by averaging the scores for each activity.

Participants also completed the Rapid Assessment of Physical Activity (RAPA) questionnaire. The RAPA is a highly sensitive self-reported questionnaire that has been validated to measure the physical activity levels of individuals over age 50 [15]. It categorizes individuals as sedentary, underactive, underactive regular-light activities, underactive regular, and active as well as assesses strength and flexibility [15].

A balance assessment was performed using the Biodex Balance System SD [16, 17]. The Biodex Balance System SD consists of a movable platform that objectively measures an individual's dynamic balance abilities. The fall risk test categorized participants as normal or above/below normal when compared to the age specified norms predetermined by the Biodex Balance SD program.

BMD of the femoral neck and spine was measured with a DEXA GE-Lunar scanner. The BMD values (in $\mathrm{g} / \mathrm{cm}^{2}$ ) for the femoral neck and L2-L4 were collected, along with Zscores and T-scores. We also noted whether an individual was osteoporotic or osteopenic or had normal bone density. One participant recently had a BMD scan independent of this study. Because only T-scores were available, this patient was excluded from most analyses, but we were able to calculate a FRAX score.

Participants were followed prospectively at the one-year mark through phone calls where information was collected on their fall history over the past year. A followup MFES was administered at this time to assess the participant's current stance on fear of falling. A modified version of the RAPA, the Telephone Assessment of Physical Activity (TAPA), was also given to evaluate current activity levels and to observe any changes in activity [18].

2.2. Statistical Analysis. Data analysis was performed using IBM SPSS Statistics for Windows, Version 21.0, Armonk, NY: IBM Corp. Data were checked for normality by examining skewness and kurtosis. Data analysis was performed for the group; the relationship between age, gender, BMI, activity level, fear of falling, and FRAX was examined. Data analyses were also disaggregated to examine gender differences. Bivariate relationships between continuous variables were determined using Pearson's correlation. Differences between categorical groups (male/female) were determined using Student's $t$-test and ANOVA and categorical variables with chi-square tests.

\section{Results}

3.1. Demographics. Of the 50 participants in this study, 17 were males and 33 were females with a mean age of $72.0 \pm 5.5$ years (range, 65-84 years) (Table 1). The average BMI was $29.3 \pm 5.8$ (range, normal-obese class III) (Table 1) [19]. The majority of participants (55\%) had a normal BMD at the 
TABLE 1: Demographic information.

\begin{tabular}{|c|c|c|c|}
\hline & Males & Females & Both \\
\hline Mean age & $73.9 \pm 5.8$ & $71.1 \pm 5.2$ & $72.0 \pm 5.5$ \\
\hline Mean BMI & $27.8 \pm 3.0$ & $30.1 \pm 6.7$ & $29.3 \pm 5.8$ \\
\hline \multicolumn{4}{|l|}{ BMI category } \\
\hline Underweight (BMI < 18.5) & 0 & 0 & 0 \\
\hline Normal (BMI 18.5-24.9) & $2(12 \%)$ & $6(18 \%)$ & $8(16 \%)$ \\
\hline Overweight (BMI 25.0-29.9) & $12(71 \%)$ & $12(36 \%)$ & $24(48 \%)$ \\
\hline Obese class I (BMI 30.0-34.9) & $2(12 \%)$ & $9(27 \%)$ & $11(22 \%)$ \\
\hline Obese class II (BMI 35.0-39.9) & $1(6 \%)$ & $3(9 \%)$ & $4(8 \%)$ \\
\hline Obese class III (BMI $\geq 40.0)$ & 0 & $3(9 \%)$ & $3(6 \%)$ \\
\hline \multicolumn{4}{|l|}{$\mathrm{BMD}$} \\
\hline Osteoporosis & 0 & $1(3 \%)$ & $1(2 \%)$ \\
\hline Osteopenia & $3(18 \%)$ & $18(56 \%)$ & $21(43 \%)$ \\
\hline Normal & $14(82 \%)$ & $13(41 \%)$ & $27(55 \%)$ \\
\hline \multicolumn{4}{|l|}{ Balance } \\
\hline Better than normal & 0 & $4(12 \%)$ & $4(8 \%)$ \\
\hline Normal & $3(18 \%)$ & $11(33 \%)$ & $14(28 \%)$ \\
\hline Poor & $14(82 \%)$ & $18(55 \%)$ & $32(64 \%)$ \\
\hline \multicolumn{4}{|l|}{ RAPA } \\
\hline Sedentary & 0 & 0 & 0 \\
\hline Underactive & 0 & 0 & 0 \\
\hline Under-active regular-light activities & $1(6 \%)$ & $8(24 \%)$ & $9(18 \%)$ \\
\hline Under-active regular & $3(18 \%)$ & $11(33 \%)$ & $14(28 \%)$ \\
\hline Active & $13(76 \%)$ & $14(42 \%)$ & $27(54 \%)$ \\
\hline \multicolumn{4}{|l|}{ FRAX } \\
\hline FRAX osteoporotic fracture (\%) & $6.12 \pm 2.83^{*}$ & $10.79 \pm 5.12^{*}$ & $9.20 \pm 4.97$ \\
\hline FRAX hip fracture (\%) & $1.88 \pm 2.11$ & $2.10 \pm 2.07$ & $2.02 \pm 2.06$ \\
\hline
\end{tabular}

TABLE 2: Correlation between age, BMI, MFES scores, and FRAX osteoporotic and hip fracture scores for both genders.

\begin{tabular}{lccccc}
\hline & Age & BMI & MFES score & FRAX osteoporotic fracture score & FRAX hip fracture score \\
\hline Age & - & - & - & - & - \\
BMI & $-0.32^{*}$ & - & - & - & - \\
MFES score & -0.15 & 0.02 & - & - & - \\
FRAX osteoporotic fracture score & $0.31^{*}$ & $-0.30^{*}$ & $-0.34^{*}$ & - & - \\
FRAX hip fracture score & $0.55^{* *}$ & $-0.39^{* *}$ & -0.24 & $0.83^{* *}$ & - \\
\hline
\end{tabular}

${ }^{*} P<0.05,{ }^{* *} P<0.01$.

femoral neck, while $43 \%$ of the sample had osteopenia (one female with osteoporosis) (Table 1).

3.2. Fear of Falling. The average MFES score was $9.47 \pm 0.97$ (best possible score 10), indicating that this cohort had little to no fear of falling. There were no significant differences in MFES scores between males $(9.69 \pm 0.59)$ and females $(9.35 \pm 1.11)(P=0.26)$. There was no significant relationship between the fear of falling and age, BMI, or FRAX ten-year probability of hip fracture scores (Table 2). There was also no significant difference in fear of falling among balance fall risk categories $(P=0.32)$ or BMD categories $(P=0.71)$ (Table 3). Males differ from the combined gender analysis and the female analysis in that there was a significant difference in fear of falling and balance fall risk categories (Table 3). The average MFES score for males with normal balance was $9.02 \pm 1.15$, and the average score for males with poor balance was $9.83 \pm 0.32$. This difference was significant $(P=0.03)$.

Males had less fear of falling with poorer balance. This was the opposite trend when compared to females, who had greater fear of falling with poorer balance. A significant correlation was found between fear of falling and FRAX tenyear probability of major osteoporotic fracture scores $(r=$ $-0.34, P=0.02$ ). When analysis of fear of falling was done within gender groups, there were a few notable differences. Fear of falling for females still had a significant and negative 
TABLE 3: MFES scores* among balance fall risk, BMD, and RAPA categories.

\begin{tabular}{lccc}
\hline & Males & Females & Both \\
\hline Balance & & & \\
$\quad$ Better than normal & 0 & $9.80 \pm 0.40$ & $9.80 \pm 0.40$ \\
Normal & $9.02 \pm 1.15^{* *}$ & $9.15 \pm 1.51$ & $9.12 \pm 1.40$ \\
Poor & $9.83 \pm 0.32^{* *}$ & $9.38 \pm 0.94$ & $9.58 \pm 0.76$ \\
BMD & & & \\
Osteoporotic & 0 & $8.86 \pm 0$ & $9.86 \pm 0$ \\
Osteopenic & $10.00 \pm 0$ & $9.27 \pm 1.17$ & $9.38 \pm 1.11$ \\
Normal & $9.62 \pm 0.64$ & $9.46 \pm 1.12$ & $9.54 \pm 0.89$ \\
RAPA & & & \\
Under-active regular-light activities & $9.00 \pm 0$ & $9.32 \pm 0.81$ & $9.28 \pm 0.77$ \\
Under-active regular & $9.02 \pm 1.15^{* *}$ & $8.97 \pm 1.72$ & $9.98 \pm 1.58^{* *}$ \\
Active & $9.90 \pm 0.22^{* *}$ & $9.68 \pm 0.38$ & $9.78 \pm 0.33^{* *}$ \\
\hline
\end{tabular}

${ }^{*}$ Higher scores indicate less fear of falling.

${ }^{* *} P<0.05$.

correlation with FRAX osteoporotic fracture scores $(r=$ $-0.36, P=0.04)$; however, this same correlation for males was nonsignificant and positive $(r=0.07, P=0.79)$.

The relationship between fear of falling and RAPA categories approached statistical significance $(P=0.07)$. In particular, the difference in fear of falling between the active and underactive regular RAPA categories was significant $(P=$ 0.03 ), with the active group having a mean MFES score of $9.78 \pm 0.33$ and the underactive regular group having a mean MFES score of $8.98 \pm 1.58$ (Table 3 ). A significant difference was found between fear of falling and RAPA categories for males $(P=0.02)$, but this difference was not significant for females $(P=0.31)$ (Table 3$)$.

3.3. Fall Risk Balance Testing. Baseline results of the fall risk balance testing showed that a majority of the sample (64\%) had poor balance (Table 1). The difference in balance between males and females was not significant $(P=0.11)$ (Table 1$)$. There was no significant relationship between balance and age, BMI, the FRAX risk of major osteoporotic fracture, or FRAX risk of hip fracture. The relationship between age and balance approached statistical significance $(P=0.06)$, with the mean ages of the better than normal, normal, and poor balance groups being $66.5 \pm 1.0,72.4 \pm 6.0$, and $72.5 \pm 5.4$ years, respectively. The relationship between BMI and balance for females also approached statistical significance $(P=0.06)$. The mean BMI of the better than normal balance group was $25.65 \pm 5.45$, the normal balance group was $26.89 \pm 2.53$, and the poor balance group was $33.12 \pm 7.45$. Activity level was not significantly impacted by balance for both genders $(P=0.69)$.

3.4. Rapid Assessment of Physical Activity. No participants were categorized as sedentary or underactive. Based on the RAPA questionnaire, the majority of the participants were categorized as active, with males more active than females (Table 1). This difference approached statistical significance $(P=0.06)$. Activity level did not have a significant relationship with age, BMI, FRAX osteoporotic fracture score, or
FRAX hip fracture score for males, females, and both genders combined.

3.5. Bone Mineral Density. Gender had a significant impact on $\operatorname{BMD}(P=0.02)$ (Table 1$)$. There was no significant relationship between BMD when compared to age and BMI. BMD had a significant relationship with both the FRAX osteoporotic fracture score $(P<0.01)$ and the FRAX hip fracture score $(P<0.01)$ for both genders combined and females. While there was a significant relationship between BMD and the FRAX osteoporotic fracture score $(P=0.03)$ for males, the relationship of BMD with the FRAX hip fracture score was not significant $(P=0.20)$.

3.6. FRAX Scores. The average ten-year probability of major osteoporotic fracture as determined by FRAX was $9.20 \% \pm$ $4.97 \%$ (range, $3.30 \%-23.00 \%$ ). Females had a significantly higher ten-year probability of major osteoporotic fracture than males $(P<0.01)$ (Table 1$)$. The ten-year probability of hip fracture based on the FRAX score was $2.02 \% \pm$ $2.06 \%$ (range, $0.00 \%-8.40 \%$ ). No significant difference was found in the ten-year probability of hip fracture between males and females $(P=0.72)$ (Table 1$)$. Age was found to have a significant and positive correlation with both FRAX scores for both genders combined and females; however, this correlation was not significant for males (Tables 2, 4, and 5). BMI, on the other hand, had a significant and negative correlation with both FRAX scores for both genders, males, and females (Tables 2, 4, and 5). The two FRAX scores had a significant and strong correlation with each other (Tables 2 , 4 , and 5).

3.7. One-Year Followup. Out of the original 50 participants in this study, 42 took part in the one-year followup (84\%). Among the eight lost to followup, we were unable to contact two participants and the other six withdrew from the study. The remaining 14 males and 28 females had a mean age of $72.4 \pm 5.7$ years (range, $66-85$ years) and a mean BMI of $29.0 \pm$ 5.7 (range, normal-obese class III) (Table 6) [19]. 
TABLE 4: Correlation between age, BMI, MFES scores, and FRAX osteoporotic and hip fracture scores for males.

\begin{tabular}{lccccc}
\hline & Age & BMI & MFES score & FRAX osteoporotic fracture score & FRAX hip fracture score \\
\hline Age & - & - & - & - & - \\
BMI & -0.44 & - & - & - & - \\
MFES score & -0.10 & -0.07 & - & - & - \\
FRAX osteoporotic fracture score & 0.42 & $-0.61^{*}$ & 0.07 & - & - \\
FRAX hip fracture score & 0.47 & $-0.61^{*}$ & -0.02 & $0.93^{*}$ & - \\
\hline
\end{tabular}

${ }^{*} P<0.01$.

TABLE 5: Correlation between age, BMI, MFES scores, and FRAX osteoporotic and hip fracture scores for females.

\begin{tabular}{lccccc}
\hline & Age & BMI & MFES score & FRAX osteoporotic fracture score & FRAX hip fracture score \\
\hline Age & - & - & - & - & - \\
BMI & -0.27 & - & - & - & - \\
MFES score & -0.24 & 0.07 & - & - & - \\
FRAX osteoporotic fracture score & $0.54^{* *}$ & $-0.43^{*}$ & $-0.36^{*}$ & - & - \\
FRAX hip fracture score & $0.64^{* *}$ & $-0.38^{*}$ & -0.31 & $0.93^{* *}$ & - \\
\hline
\end{tabular}

${ }^{*} P<0.05,{ }^{* *} P<0.01$.

The average MFES score at one-year followup was $9.35 \pm$ 0.94. Consistent with our initial results, no significant difference was found between fear of falling for males $(9.47 \pm$ $1.09)$ and females $(9.29 \pm 0.86)(P=0.56)$. The trend that older males are more active than older females was found to be significant at one-year followup $(P<0.01)$ (Table 6). However, the activity level was not found to have a significant association with age $(P=0.61)$, BMI $(P=0.13)$, or fear of falling $(P=0.86)$.

Eight individuals (two males, six females) experienced a fall in the previous year, with one female participant experiencing multiple falls. On initial testing, five of these participants had poor balance when compared to the norms for their age group. Two female participants had normal balance, and one female participant had better than normal balance. Average FRAX score for major osteoporotic fracture was $11.51 \% \pm 5.58 \%$ and the average FRAX score for hip fracture was $2.88 \% \pm 2.38 \%$. Three participants had a normal BMD (one male, two females), four were osteopenic (one male, three females), and one female was osteoporotic. These individuals also became more afraid of falling, with an initial average MFES score of $9.23 \pm 1.44$ decreasing to $9.11 \pm 1.12$ one-year later, though this was not a significant change $(P=$ $0.83)$. They also became less active, though again the change from baseline to the one-year point was not significant $(P=$ 0.48). Initially seven individuals were considered active with one female participant being underactive regular. After oneyear, only three individuals were still active (one male, two females), with four being underactive regular (one male, three females) and one female being underactive.

Seven participants did not sustain any injuries due to their falls, except for one female participant who suffered a fractured foot and subsequently required the use of a walker to ambulate. The female participant who suffered a fracture was one of the oldest participants in the study at 84 years old. Prior to her fragility fracture she had been active. She was osteopenic with poor balance and FRAX scores of $15.00 \%$ and
$4.10 \%$ for osteoporotic and hip fractures, respectively. After her fragility fracture she became much more afraid of falling, with her MFES score decreasing from 9.54 to 7.64 .

\section{Discussion}

The participants in this study consisted of ambulatory community-dwelling volunteers. They were generally healthy and physical active, with no sedentary individuals. Nearly half were overweight and one had osteoporosis, and they had little to no fear of falling and were fairly healthy and active; however, their balance scores were generally poor. In addition, the difference in balance between males and females approached statistical significance $(P=0.11)$, which may suggest a trend that older males have poorer balance than older females. The overall poor balance is an unexpected finding because physically activity is believed to improve balance and reduce the risk of falls $[9,20,21]$. We did not find a significant relationship between balance and activity level and fall and fracture risk; however, this may be due to our small sample size. This finding is of concern because it suggests that many older adults are unaware of their capability with respect to balance. Therefore, balance screening and training is important even in active healthy nonosteoporotic individuals to decrease future fall and fracture risk.

Our finding of no difference in fear of falling between males and females contradicts the literature. Several studies examining fear of falling in community-dwelling seniors have shown that women are significantly more afraid of falling than men [22-24]; however, our findings may have been influenced by a small sample size and selection bias. Although we did not find a gender difference, fear of falling can have serious implications. Our cohort overall had very little fear of falling; even the lower MFES scores that were recorded for this sample are considered to have little fear of falling. However, even between the small range of scores significant differences were found. 
TABLE 6: One-year followup data on demographics and TAPA* categories.

\begin{tabular}{lccc}
\hline & Males & Females & Both \\
\hline Mean age & $74.8 \pm 6.0$ & $71.1 \pm 5.3$ & $72.4 \pm 5.7$ \\
Mean BMI & $27.6 \pm 2.9$ & $29.7 \pm 6.7$ & $29.0 \pm 5.7$ \\
BMI category & & & 0 \\
$\quad$ Underweight (BMI $<$ 18.5) & 0 & 0 & $10(24 \%)$ \\
Normal (BMI 18.5-24.9) & $2(14 \%)$ & $8(29 \%)$ & $17(40 \%)$ \\
Overweight (BMI 25.0-29.9) & $10(72 \%)$ & $7(25 \%)$ & $9(21 \%)$ \\
Obese class I (BMI 30.0-34.9) & $1(7 \%)$ & $8(29 \%)$ & $4(10 \%)$ \\
Obese class II (BMI 35.0-39.9) & $1(7 \%)$ & $3(10 \%)$ & $2(5 \%)$ \\
Obese class III (BMI $\geq 40.0)$ & 0 & $2(7 \%)$ & $11(26 \%)$ \\
TAPA & & & $13(31 \%)$ \\
Under-active & 0 & $11(39 \%)$ & $18(43 \%)$ \\
Under-active regular & $4(29 \%)$ & $8(32 \%)$ & \\
Active & $10(71 \%)^{* *}$ & & \\
\hline
\end{tabular}

* Telephone assessment of physical activity [18].

${ }^{* *} P<0.01$.

It has been well documented in the literature that osteoporosis is more prevalent in women than in men [25-27]. Therefore, our finding that gender significantly impacts BMD was expected. This also likely contributed to our finding that females had a significantly greater risk of osteoporotic fracture, as determined by FRAX scores. As well, considering that BMD is one of the components used to calculate FRAX scores, it was no surprising that BMD had a strong relationship with both the FRAX osteoporotic fracture score and the FRAX hip fracture score.

$\mathrm{BMI}$ is another risk factor that has been shown to have some influence on fragility fractures [7, 28]. A low BMI is believed to increase fracture risk because there is less soft tissue protecting the bones $[7,28]$. Therefore, our finding of a negative relationship between BMI and FRAX is expected as a higher BMI would be expected to reduce fracture risk as the individual would have more soft tissue.

Individuals who have a greater fear of falling have a significantly greater risk of sustaining a major osteoporotic fracture based on the FRAX score. There are a number of potential reasons that may explain this. It may be that individuals with a poor BMD have some awareness of their bone health issues either because these have been previously addressed by health professionals or because they have health problems or symptoms that initiate concern and as a result have a greater fear of falling. Since BMD has a strong association with osteoporotic fracture risk [26, 27, 29, 30], this appears like a plausible explanation. However, fall and fracture risk is multifactorial and does not rely solely on $\operatorname{BMD}[5,8,11,12,30]$. The relationship between fear of falling and BMD was not significant, suggesting that the increased fracture risk in the more fearful individuals was influenced by risk factors other than BMD (i.e., other factors used to calculate FRAX scores such as previous fracture, parent with a fractured hip, smoking and excessive alcohol consumption, long-term glucocorticoid use, rheumatoid arthritis, and causes of secondary osteoporosis) [11-13].
Another factor influencing fall and fracture risk is activity level. It is believed that decreasing activity levels leads to a functional decline, predisposing individuals to fall $[2,9,10]$. Fear of falling is a common reason for older individuals to decrease their activity level $[2,10,22,31-33]$, which supports our finding that more active individuals have less fear of falling. Individuals who suffered a fall at the one-year followup had experienced some increased fear of falling and decreased activity level compared to those who did not fall. In addition, our findings suggest that fear of falling affects activity levels of males more than females. Balance is also believed to be closely related to activity level and fall and fracture risk [20], but we did not find a significant relationship between these factors. Even though we found an association between fear of falling and activity level, with fear of falling known to influence osteoporotic fracture risk, we did not find a relationship between activity level and fracture risk.

The individuals who experienced a fall at one-year followup had higher FRAX scores for major osteoporotic fracture and hip fracture than those who did not fall. As well, many of the individuals who fell experienced a decrease in their activity level and became more afraid of falling, whereas individuals who did not fall did not experience much change in their activity or fear of falling. This supports the notion that falls, even if they do not cause injury, increase fear of falling, and can lead to decreased activity and potentially a functional decline in older adults [7, 14, 22, 23]. Although specific measures to improve or maintain activity level were not evaluated in this study, encouraging older adults to stay active should help minimize their fear of falling and falls. Future studies are needed to assess specific activities that will help maintain balance and prevent falls.

The results of this study may be influenced by selection bias since the cohort consisted of healthy volunteers. Another limitation is that the number of males who participated was small; therefore, conclusions based around the male gender are not as robust as the conclusions for females. As well, 
the one-year followup relied upon accurate reporting by the participants. Considering that there was no contact between the researchers and the study participants over a period of 12 months, it is possible that recall bias influenced fall reporting. We might have been able to obtain a more precise picture of fall risk with more regular followup with the participants. In spite of these limitations, this study was able to successfully follow a cohort longitudinally over one-year. In doing this, we were able to identify the differences in risk factors between individuals who did and did not suffer a fall.

\section{Conclusions}

Our baseline data showed that individuals with greater fear of falling had an increased fracture risk. The finding that overall balance was poor in this healthy, active population was unexpected. More attention should be dedicated towards balance interventions in this population to minimize fall risk. Individuals who fell after one-year experienced an increase in their fear of falling as well as decreased activity levels. This emphasizes the need for methods to reduce fear of falling in active healthy seniors in order to prevent future falls, fractures, and functional decline.

\section{Abbreviations}

BMI: Body mass index

BMD: Bone mineral density

DEXA: Dual-energy X-ray absorptiometry

FRAX: Fracture Risk Assessment Tool

MFES: Modified Falls Efficacy Scale

RAPA: Rapid Assessment of Physical Activity

TAPA: Telephone Assessment of Physical Activity

WHO: World Health Organization.

\section{Acknowledgment}

This work was supported by an operating grant received from the Canadian Institute of Health Research (CIHR) under project titled Identification of Risk of Adverse Activity Transtition following a Distal Radius Fracture. PIs are MacDermid JC and Grewal R and Coinvestigators are McIntyre $\mathrm{N}$ and Richardson J. Award no. MOP-93372.

\section{References}

[1] V. Scott, M. Pearce, and C. Pengelly, "Technical report: hospitalizations due to falls among Canadians age 65 and over," Tech. Rep. HP25-1/20005E, Public Health Agency of Canada, Ottawa, Canada, 2005.

[2] J. A. Stevens, P. S. Corso, E. A. Finkelstein, and T. R. Miller, "The costs of fatal and non-fatal falls among older adults," Injury Prevention, vol. 12, no. 5, pp. 290-295, 2006.

[3] S. Heinrich, K. Rapp, U. Rissmann, C. Becker, and H.-H. König, "Cost of falls in old age: a systematic review," Osteoporosis International, vol. 21, no. 6, pp. 891-902, 2010.

[4] K. Malik, "Development Report 2013," Tech. Rep., United Nations Development Programme, New York, NY, USA, 2013.
[5] Canadian Demographics at a Glance, Catalogue 91-003-X, Statistics Canada, Ottawa, Canada, 2008.

[6] M. E. Tinetti, M. Speechley, and S. F. Ginter, "Risk factors for falls among elderly persons living in the community," The New England Journal of Medicine, vol. 319, no. 26, pp. 1701-1707, 1988.

[7] S. D. Berry and R. R. Miller, "Falls: epidemiology, pathophysiology, and relationship to fracture," Current Osteoporosis Reports, vol. 6, no. 4, pp. 149-154, 2008.

[8] National Trauma Registry 2011 Report: Hospitalizations for Major Injury in Canada, 2008-2009 Data, Toronto, Canada, Canadian Institute for Health Information, 2011.

[9] C. M. Arnold, M. M. Sran, and E. L. Harrison, "Exercise for fall risk reduction in community-dwelling older adults: a systematic review," Physiotherapy Canada, vol. 60, pp. 358-372, 2008.

[10] D. G. Bruce, A. Devine, and R. L. Prince, "Recreational physical activity levels in healthy older women: the importance of fear of falling," Journal of the American Geriatrics Society, vol. 50, no. 1, pp. 84-89, 2002.

[11] J. A. Kanis, E. V. McCloskey, H. Johansson, A. Oden, O. Ström, and F. Borgström, "Development and use of FRAX in osteoporosis," Osteoporosis International, vol. 21, supplement 2, pp. S407-S413, 2010.

[12] N. B. Watts, "The fracture risk assessment tool (FRAX): applications in clinical practice," Journal of Women's Health, vol. 20, no. 4 , pp. 525-531, 2011.

[13] A. Papaioannou, S. Morin, A. M. Cheung et al., "2010 clinical practice guidelines for the diagnosis and management of osteoporosis in Canada: summary," Canadian Medical Association Journal, vol. 182, no. 17, pp. 1864-1873, 2010.

[14] K. D. Hill, J. A. Schwarz, A. J. Kalogeropoulos, and S. J. Gibson, "Fear of falling revisited," Archives of Physical Medicine and Rehabilitation, vol. 77, no. 10, pp. 1025-1029, 1996.

[15] T. D. Topolski, J. LoGerfo, D. L. Patrick, B. Williams, J. Walwick, and M. B. Patrick, "The Rapid Assessment of Physical Activity (RAPA) among older adults," Preventing Chronic Disease, vol. 3, no. 4, p. A118, 2006.

[16] K. Y. Oh, S. A. Kim, S. Y. Lee et al., "Comparison of manual balance and balance board tests in healthy adults," Annals of Physical and Rehabilitation Medicine, vol. 35, pp. 873-879, 2011.

[17] M. L. Pickerill and R. A. Harter, "Validity and reliability of limits-of-stability testing: a comparison of 2 postural stability evaluation devices," Journal of Athletic Training, vol. 46, no. 6, pp. 600-606, 2011.

[18] C. J. Mayer, L. Steinman, B. Williams, T. D. Topolski, and J. LoGerfo, "Developing a Telephone Assessment of Physical Activity (TAPA) questionnaire for older adults," Preventing Chronic Disease, vol. 5, no. 1, p. A24, 2008.

[19] Health Canada, "Description of the Canadian Guidelines for Body Weight Classification in Adults," 2003, http://www.hcsc.gc.ca/fn-an/nutrition/weights-poids/guide-ld-adult/weight book-livres_des_poids-03-tablel-eng.php.

[20] A. Barnett, B. Smith, S. R. Lord, M. Williams, and A. Baumand, "Community-based group exercise improves balance and reduces falls in at-risk older people: a randomised controlled trial," Age and Ageing, vol. 32, no. 4, pp. 407-414, 2003.

[21] L. Clemson, M. A. Fiatarone Singh, A. Bundy et al., "Integration of balance and strength training into daily life activity to reduce rate of falls in older people (the LiFE study): randomised parallel trial," British Medical Journal, vol. 345, Article ID e4547, 2012.

[22] R. Boyd and J. A. Stevens, "Falls and fear of falling: burden, beliefs and behaviours," Age and Ageing, vol. 38, no. 4, pp. 423428, 2009. 
[23] H. W. Lach, "Incidence and risk factors for developing fear of falling in older adults," Public Health Nursing, vol. 22, no. 1, pp. 45-52, 2005.

[24] G. A. R. Zijlstra, J. C. M. van Haastregt, J. T. M. van Eijk, E. van Rossum, P. A. Stalenhoef, and G. I. J. M. Kempen, "Prevalence and correlates of fear of falling, and associated avoidance of activity in the general population of community-living older people," Age and Ageing, vol. 36, no. 3, pp. 304-309, 2007.

[25] P. M. Cawthon, "Gender differences in osteoporosis and fractures," Clinical Orthopaedics and Related Research, vol. 469, no. 7, pp. 1900-1905, 2011.

[26] N. E. Lane, "Epidemiology, etiology, and diagnosis of osteoporosis," American Journal of Obstetrics and Gynecology, vol. 194, no. 2, supplement, pp. S3-S11, 2006.

[27] NIH Consensus Development Panel on Osteoporosis Prevention, Diagnosis, "Osteoporosis prevention, diagnosis, and therapy," Journal of the American Medical Association, vol. 285, pp. 785-795, 2001.

[28] C. De Laet, J. A. Kanis, A. Odén et al., "Body mass index as a predictor of fracture risk: a meta-analysis," Osteoporosis International, vol. 16, no. 11, pp. 1330-1338, 2005.

[29] O. Johnell, J. A. Kanis, A. Oden et al., "Predictive value of BMD for hip and other fractures," Journal of Bone and Mineral Research, vol. 20, pp. 1185-1194, 2005.

[30] J. A. Kanis, "Osteoporosis III: diagnosis of osteoporosis and assessment of fracture risk," The Lancet, vol. 359, no. 9321, pp. 1929-1936, 2002.

[31] D. Rand, W. C. Miller, J. Yiu, and J. J. Eng, "Interventions for addressing low balance confidence in older adults: a systematic review and meta-analysis," Age and Ageing, vol. 40, no. 3, pp. 297-306, 2011.

[32] G. I. J. M. Kempen, J. C. Van Haastregt, K. J. McKee, K. Delbaere, and G. R. Zijlstra, "Socio-demographic, health-related and psychosocial correlates of fear of falling and avoidance of activity in community-living older persons who avoid activity due to fear of falling," BMC Public Health, vol. 9, article 170, 2009.

[33] H. Shimada, S. R. Lord, H. Yoshida, H. Kim, and T. Suzuki, "Predictors of cessation of regular leisure-time physical activity in community-dwelling elderly people," Gerontology, vol. 53, no. 5, pp. 293-297, 2007. 


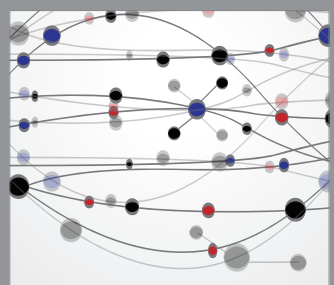

The Scientific World Journal
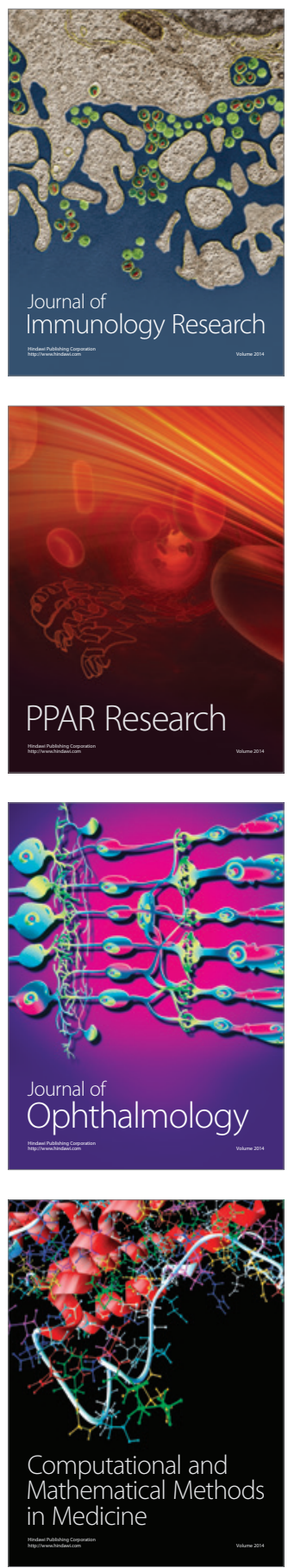

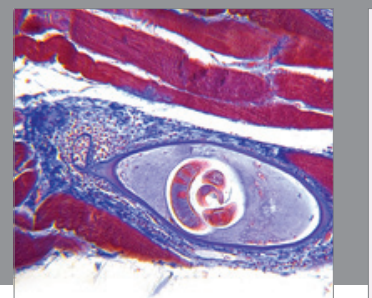

Gastroenterology

Research and Practice
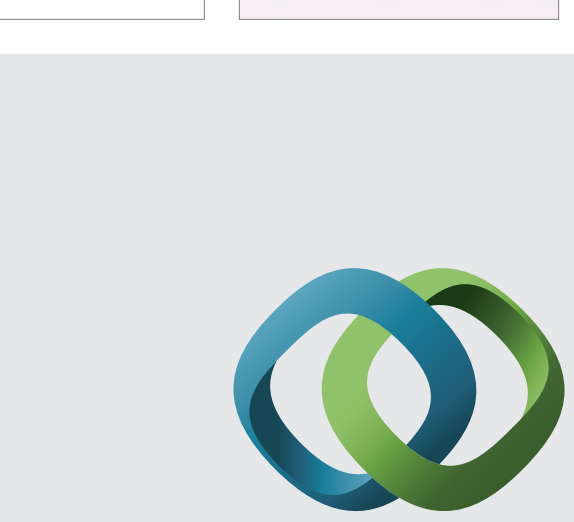

\section{Hindawi}

Submit your manuscripts at

http://www.hindawi.com
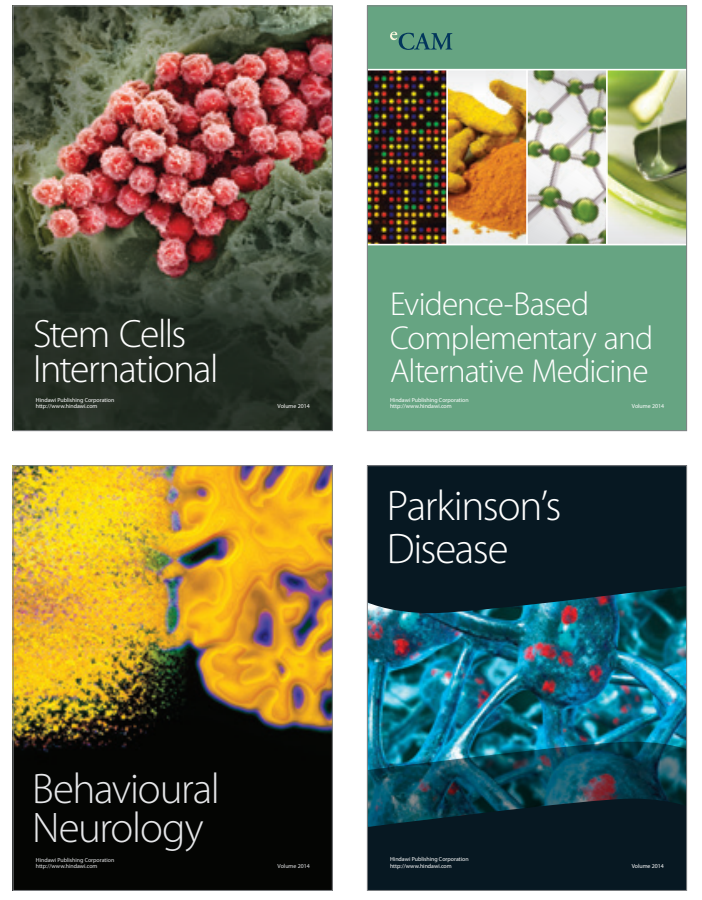
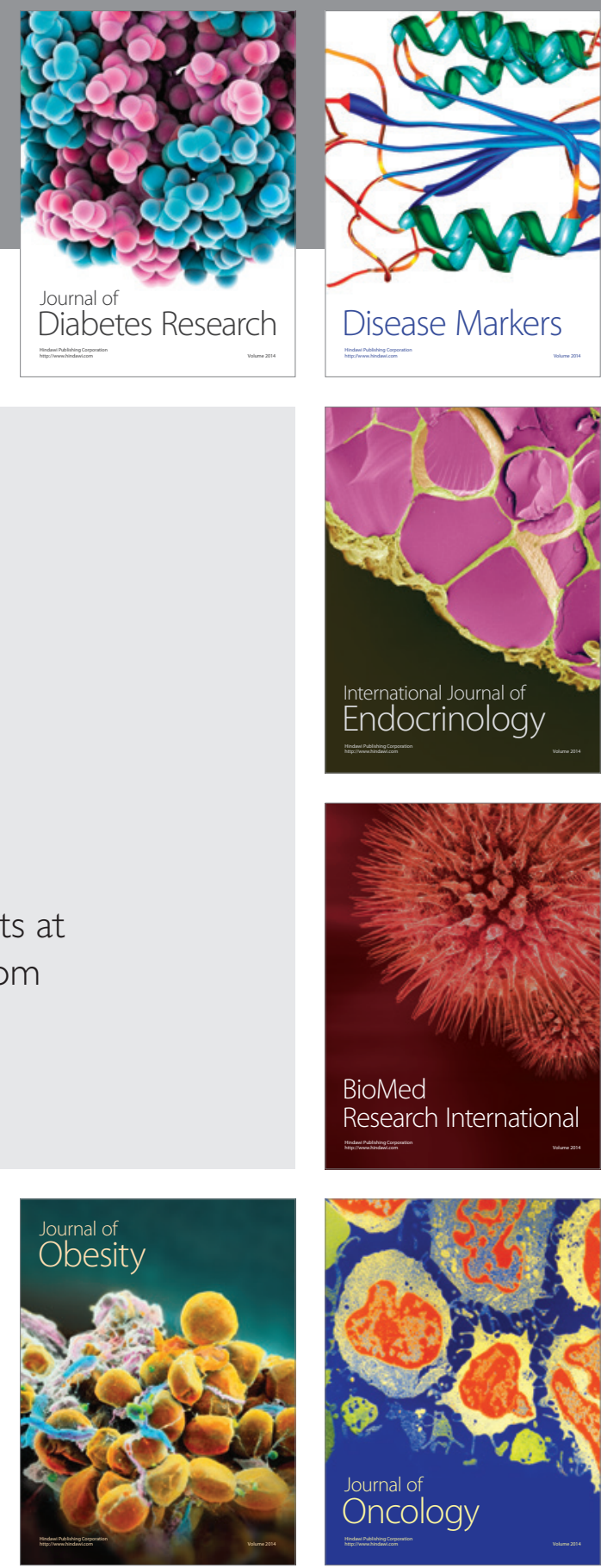

Disease Markers
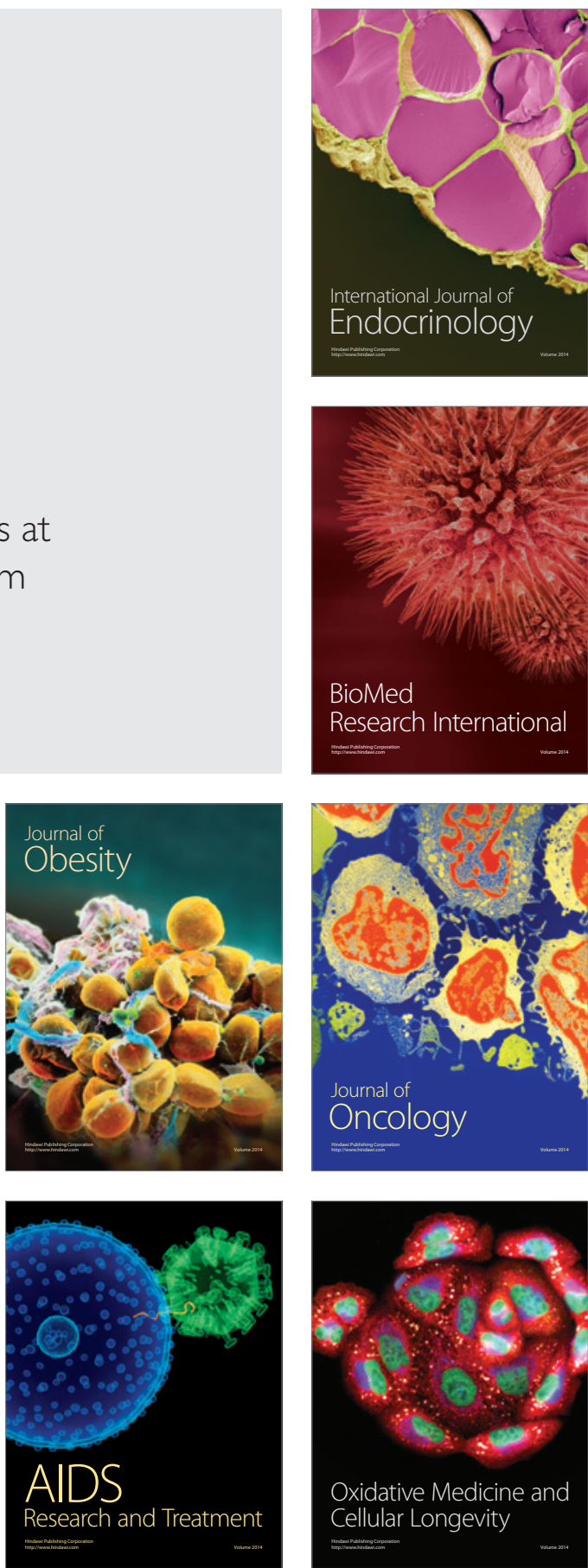